\title{
Improving grasping forces during the manipulation of unknown objects
}

\author{
Andrés Montaño and Raúl Suárez
}

\begin{abstract}
Many of the solutions proposed for the object manipulation problem are based on the knowledge of the object features. The approach proposed in this paper intends to provide a simple geometrical approach to securely manipulate an unknown object based only on tactile and kinematic information. The tactile and kinematic data obtained during the manipulation is used to recognize the object shape (at least the local object curvature), allowing to improve the grasping forces when this information is added to the manipulation strategy. The approach has been fully implemented and tested using the Schunk Dexterous Hand (SDH2). Experimental results are shown to illustrate the efficiency of the approach.
\end{abstract}

\section{INTRODUCTION AND RELATED WORK}

Object manipulation is a relevant open problem in robotics. Many approaches has been proposed to address this topic but in many cases they must be still transferred to a realistic scenario where the environment and the manipulated object are unknown. The inclusion of tactile sensors in the design of robotic hands [1] helps to obtain information about the contact with the manipulated object, potentially increasing the robot capabilities. Besides, tactile information is useful in applications where other information sources, as, for instance artificial vision, are not available [2]. In general, the tactile information is a good complement to reduce uncertainty and to approach the robotic applications to the real world [3]. The most common goals of the object manipulation are the optimization of: a) the hand configuration looking for a comfortable configuration that increases the manipulability, b) the grasp looking for a hand configuration that increases the robustness of the grasp, and c) the object configuration according to a desired one [4].

Most related works focus on control schemes but they generally do not consider tactile feedback for the object manipulation. For instance, a position control scheme was proposed to change the pose of the manipulated object [5], but the lack of sensory feedback limits the applicability of the approach. A position-force control scheme combined with a finger gaiting strategy was also proposed to manipulate an object [6], but the evaluation was performed only in simulation, introducing noise on the sensor measurements to emulate a real environment. Another approach uses a torque controller to optimize the directions of the grasping forces applied on a known object [7], the approach could manipulate objects with different shapes if tactile sensors are considered, but the experimental results were performed without them. Besides, approaches based on reinforcement

The authors are with the Institute of Industrial and Control Engineering (IOC) - Universitat Politècnica de Catalunya (UPC), Barcelona, Spain (raul.suarez@upc.edu). This work was partially supported by the Spanish Government through the project DPI2016-80077-R. learning has been proposed as an alternative to the use analytic models, dealing with problems like force estimation [8], slip detection, and manipulation of unknown objects [9].

The exploration of the object surface was addressed using, for instance, a position-force controller to control sliding movements of the fingers [10], and a rolling strategy to identify the object shape and plan the manipulation movements [11]. In a previous work, we proposed an approach to recognize the object shape based on the contact information obtained during the manipulation [12], which is used to compute distance invariants that define a signature of the manipulated object. The approach proposed in this work provides a simple geometrical procedure able to combine the recognition of the local curvature of the object surface at the contacted regions (based on information generated during the manipulation), with the manipulation strategy itself, allowing in this way an optimization of the contact forces during the manipulation process.

The paper is organized as follows. Section II presents the problem statement. Section III introduces the proposed manipulation approach, discussing the grasp modeling, the main manipulation algorithm and the object recognition procedure. Experimental results are described in Section IV. Finally, the summary and future work are presented in Section V.

\section{Problem Statement}

The problem addressed in this work is the force optimization when an unknown object is manipulated, the expression "unknown object" means that the shape of the object is not known when the manipulation starts. The manipulation is performed using two fingers of a robotic hand, the fingertips have tactile sensors that allow the measurement the contact locations and the forces applied on the manipulated object. The two fingers perform a grasp comparable with a human grasp using the thumb and index fingers. The movements of the fingertips are limited to a plane, but they allow the execution of multiple every-day and industrial tasks, as, for instance, matching the orientation of two pieces to be assembled or inspecting an object [13]. Determining the initial grasp is outside the scope of this work but it can be done using any grasp planner [14] or even just by trial and error. Here, the initial grasp is considered as a blind grasp that does not have associated information as the contact locations on the object surface, the object mass, the object center of mass, the friction coefficient, or any external force disturbances [15]. Therefore, the system only has information about the positions of the finger joints provided by the hand internal sensors and the contact points and forces on the fingertips provided by the tactile sensors. No other feedback 
sources are available, as, for instance, an external vision system to recognize the object location and shape. The friction coefficient is assumed to be above a minimum value, considering the rubber surface of the fingertips.

The goal of the proposed approach is, after performing a blind force-closure grasp of an unknown object, to iteratively determine a sequence of hand configurations that allows the secure object manipulation and, at the same time, to get information of the object shape in order to improve the contact forces during the manipulation. The computation of the manipulation movements, including the local curvatures used to improve the manipulation forces, is iteratively done using only the tactile feedback and the kinematic information of the hand obtained during the manipulation process.

\section{PROPOSED APPROACH}

\section{A. Grasp Modeling}

The geometric model of a two-finger grasp is shown in Fig. 1. Each finger $f_{i}, i \in\{1,2\}$, is a kinematic chain with $n_{i}$ links of length $l_{i j}, j \in\left\{1, . ., n_{i}\right\}$ being $n_{i}$ the number of degrees of freedom (dof) of each finger. The relative position of each link with respect to the previous one is denoted by the joint angle $q_{i j}$. A finger configuration is given by the set of joint angles $\boldsymbol{q}_{i}=\left\{q_{i 1}, \cdots, q_{i n_{i}}\right\}$. A hand configuration is the concatenation of the configurations of the two fingers $\boldsymbol{Q}=\left\{\boldsymbol{q}_{1}, \boldsymbol{q}_{2}\right\}$. The base of each finger link has an associated reference frame $\Sigma_{i j}$, and the position of the origin of $\Sigma_{i j}$ is denoted by the point $P_{\Sigma_{i j}}$. The absolute reference frame $\mathcal{W}$ is located at the base of the finger $f_{1}$. Let $P_{i}$ be the position of the contact point on finger $f_{i}$ with respect to $\mathcal{W}$. The tactile sensor measurements are referred to a sensor reference frame $\mathcal{S}$. In order to include the information provided by the tactile sensor into the hand kinematics a virtual link is used, which adds a non-controllable extra $d o f, q_{c_{i}}$, to each finger. The length $r_{i}$ of the virtual link is the segment between the origin of the reference frame $\Sigma_{i n_{i}}$, and the contact point $P_{i}^{s}$, where the subscript $s$ means that $P_{i}$ is expressed in the sensor reference frame $\mathcal{S}$. The contact points $P_{i}$ are computed using forward kinematics (FK) of the fingers and the information of the virtual link provided by the tactile sensor. The distance $d$ is the Euclidean distance between the contact points $P_{1}$ and $P_{2}$,

$$
d\left(P_{1}, P_{2}\right)=\sqrt{\left(P_{1_{x}}-P_{2_{x}}\right)^{2}+\left(P_{1_{y}}-P_{2_{y}}\right)^{2}}
$$

\section{B. Manipulation Algorithm}

Algorithm 1 shows the main steps of the manipulation procedure. The desired contact force $F_{d}$ and the desired object orientation $\gamma_{d}$ relative to the initial object orientation are the algorithm inputs. The manipulation begins once the object has been properly grasped with a force-closure grasp. The manipulation procedure iteratively computes the target hand configurations to rotate the object towards the desired orientation. In order to improve the contact forces, the manipulation is divided into two stages, in the first, the fingertip positions are computed on a single virtual

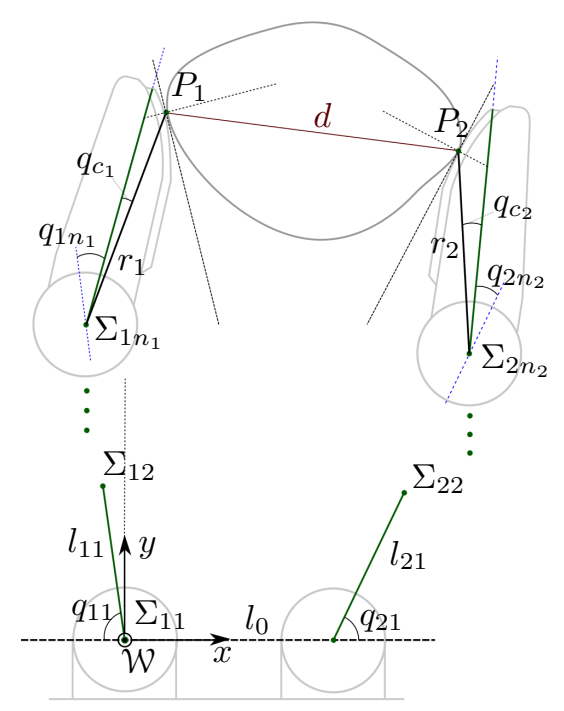

Fig. 1. Geometric model of a two-finger grasp.

circumference, while, in the second, each fingertip follows a specific circumference computed according to the local curvature of the object at the contact point. These two specific circumferences are computed using a circular regression over the set of resulting contact points of previous manipulation iterations, thus, the second stage can start only after $m$ iterations, being $m$ the number of points used to properly compute a circular regression. The local curvatures contain information about the object shape and the resulting target hand configurations using them improve the force applied on the manipulated object. Each manipulation iteration $k$ involves the following parts:

- The computation of the current state of the grasp (lines 3 to 7 ).

- The computation of the two virtual contact points $P_{1_{k+1}^{*}}^{*}$ and $P_{2_{k+1}}^{*}$, which are used as auxiliary points in the computation of target contact points $P_{1_{k+1}}$ and $P_{2_{k+1}}$ (line 9 or 12).

- The adjustment of the distance $d_{k}$ between contact points to compensate the contact force (line 13).

- The computation of the target contact points $P_{i_{k+1}}$ (line 14).

- The computation of the target hand configuration $\boldsymbol{Q}_{k+1}$ (line 15).

- The verification of the termination conditions (line 16).

- Finally, the movement of the fingers to a target configuration is performed if none of the termination conditions is activated (line 17).

The computation of the current state of the grasp implies the computation of the position of the contact points $P_{1_{k}}$ and $P_{2_{k}}$, the magnitude of the grasping force $F_{k}$, the orientation of the object $\gamma_{k}$, and the orientation error $\varepsilon_{o} . P_{1_{k}}$ and $P_{2_{k}}$ are computed using FK and the virtual link information. $F_{k}$ is the average of the contact forces $F_{1_{k}}$ and $F_{2_{k}}$ measured on each fingertip. The average of both measured contact forces is used to minimize potential measurement errors. The value of 


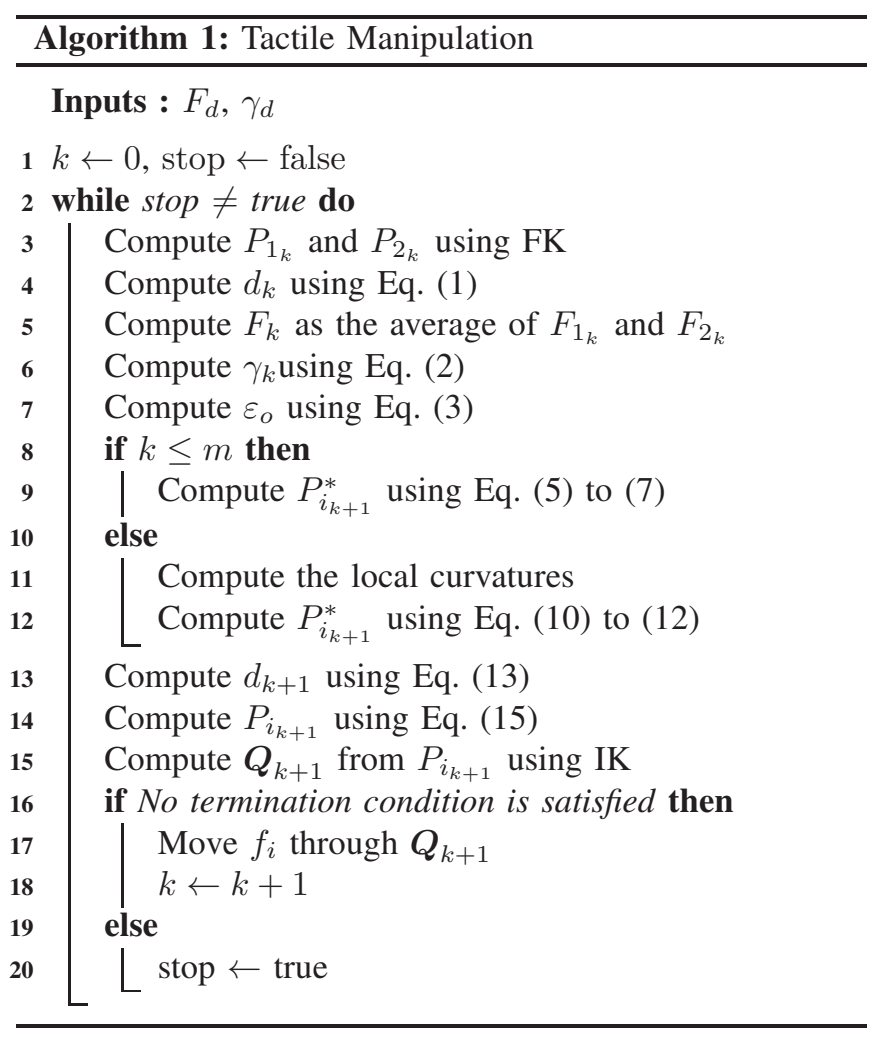

$\gamma_{k}$ in the initial grasp is considered as $\gamma_{0}=0$, and it is used as reference for the object orientation. During the iterative process, $\gamma_{k}$ is computed using the information obtained from the tactile sensors and the finger kinematics. For fingertips with circular shape, the current object orientation $\gamma_{k}$ can be approximated by [16],

$$
\gamma_{k} \approx \frac{R}{d_{k}} \sum_{j=1}^{n_{1}}\left(q_{1 j_{\gamma_{0}}}-q_{1 \mathrm{j}_{k}}\right)-\sum_{j=1}^{n_{2}}\left(q_{2 \mathrm{j}_{\gamma_{0}}}-q_{2 j_{k}}\right)
$$

The goal of the manipulation is to reduce the orientation error $\varepsilon_{o}$ between the desired orientation $\gamma_{d}$ and the current object orientation $\gamma_{k}$, i.e.

$$
\varepsilon_{o}=\gamma_{d}-\gamma_{k}
$$

During the first $m$ iterations there is no information about the object shape, therefore the two virtual contact points $P_{1_{k+1}}^{*}$ and $P_{2_{k+1}}^{*}$ are computed considering them as the result of a displacement of the current contact points on the sensor pad along a virtual circular path with diameter $d_{k}$, centered at the middle point $R_{k}$ between the points $P_{1_{k}}$ and $P_{2_{k}}$, i.e. the virtual contact points are given by (see Figure 2):

$$
\begin{aligned}
& P_{1_{k+1}}^{*}=R_{k_{x}}-\left(d_{k} / 2\right) \cos \left(\gamma_{k+1}\right) \\
& P_{1_{k+1}}^{*}=R_{k_{y}}-\left(d_{k} / 2\right) \sin \left(\gamma_{k+1}\right) \\
& P_{2_{k+1}}^{*}=R_{k_{x}}+\left(d_{k} / 2\right) \cos \left(\gamma_{k+1}\right) \\
& P_{2_{k+1}}^{*}=R_{k_{y}}+\left(d_{k} / 2\right) \sin \left(\gamma_{k+1}\right)
\end{aligned}
$$

with

$$
\gamma_{k+1}=\gamma_{k}+\tanh \left(\varepsilon_{o}\right) \Delta \gamma
$$

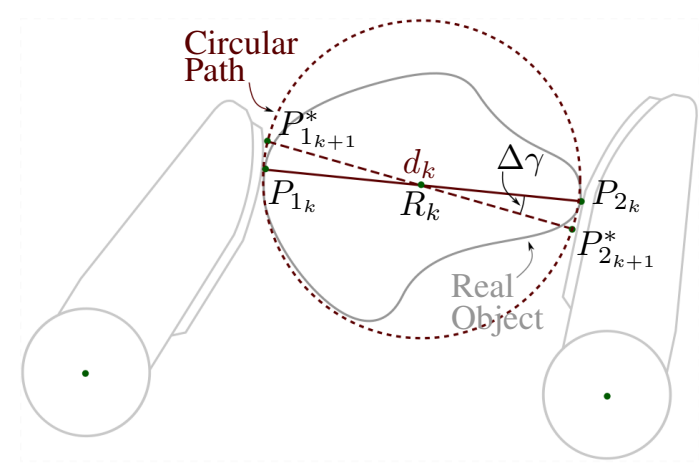

Fig. 2. Movements used to change the object orientation. $P_{1_{k+1}^{*}}^{*}$ and $P_{2_{k+1}}^{*}$ are computed over a circular path with diameter $d_{k}$ centered at $R_{k}$.

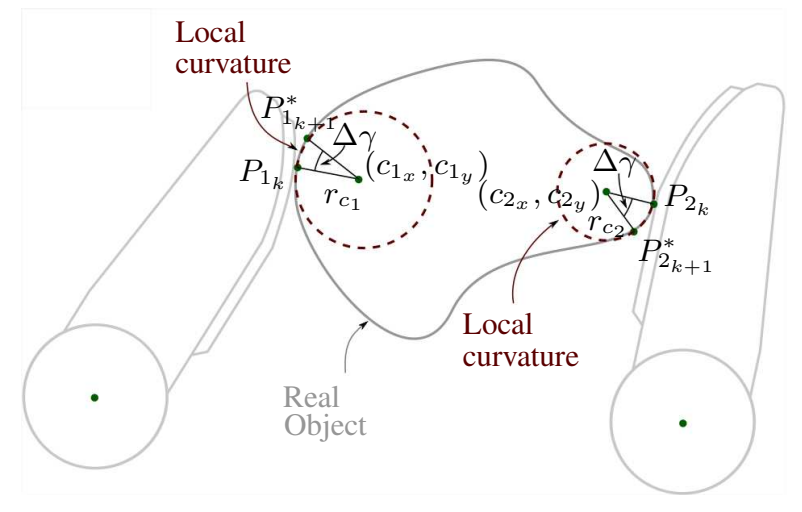

Fig. 3. Movements used to change the object orientation improving the contact force. $P_{1_{k+1}}^{*}$ and $P_{2_{k+1}}^{*}$ are computed over the circular paths generated by the circular regressions using the previous contact points.

where $\Delta \gamma>0$ is chosen empirically and small enough to assure small movements of the object in each iteration and the function tanh is used to limit the gain for large values of $\gamma_{k}$. Note that the point $R_{k}$ is recomputed in each iteration.

On the contrary, after $m$ iterations (i.e. $k>m$ ), $P_{1_{k+1}}^{*}$ and $P_{2_{k+1}}^{*}$ are computed considering them as the result of a displacement of the current contact points on the sensor pad along circular paths described by the circumferences $C_{i}$ with center at $\left(c_{i_{x}}, c_{i_{y}}\right)$ and radius $r_{c_{i}}$, obtained using the circular regression as described below in Section III-C, i.e. the virtual circular path is replaced by paths described by the circumferences computed using the data obtained during the manipulation (see Figure 3 ). Therefore, $P_{1_{k+1}^{*}}^{*}$ and $P_{2_{k+1}^{*}}^{*}$ are given by,

$$
\begin{aligned}
& P_{1_{k+1}}^{*}=c_{1_{x_{k}}}-\left(r_{c_{1_{k}}}\right) \cos \left(\gamma_{k+1}\right) \\
& P_{1_{k+1}}^{*}=c_{1_{y_{k}}}-\left(r_{c_{1_{k}}}\right) \sin \left(\gamma_{k+1}\right) \\
& P_{2_{k+1}}^{*}=c_{2_{x_{k}}}+\left(r_{c_{2_{k}}}\right) \cos \left(\gamma_{k+1}\right) \\
& P_{2_{k+1_{y}}^{*}}^{*}=c_{2_{y_{k}}}+\left(r_{c_{2_{k}}}\right) \sin \left(\gamma_{k+1}\right)
\end{aligned}
$$

In both cases, the movements of the fingers change the contact force $F_{k}$. If $F_{k}$ increases could produce a damage of the object or the hand, and if $F_{k}$ decreases could produce a fall of the object. In order to reduce the error $\varepsilon_{f}=F_{k}-F_{d}$, 


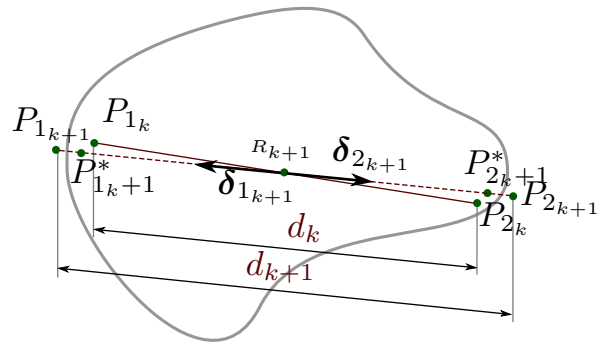

Fig. 4. Example of computation of $P_{i_{k+1}}$ using $P_{i_{k+1}}^{*}$ adjusting the distance $d_{k}$ to $d_{k+1}$ when the contact force $F_{k}$ is larger than $F_{d}$.

the distance $d_{k}$ is adjusted in each iteration as

$$
d_{k+1}=d_{k}+\Delta d
$$

with

$$
\Delta d= \begin{cases}2 \lambda\left(\varepsilon_{f}+\varepsilon_{f}^{2}\right) & \text { if } \varepsilon_{f} \leq 0 \\ \lambda \varepsilon_{f} & \text { if } \varepsilon_{f}>0\end{cases}
$$

where $\lambda>0$ is a predefined constant. The reason for this adjustment is that a potential fall of the object $\left(F_{k} \rightarrow 0\right)$ is considered more critical that a potential application of large grasping forces $\left(F_{k} \gg F_{d}\right)$.

Now, the virtual contact points $P_{1_{k+1}}^{*}$ and $P_{2_{k+1}}^{*}$ are adjusted to obtain the actual target contact points $P_{1_{k+1}}$ and $P_{2_{k+1}}$ separated by a distance $d_{k+1}$,

$$
P_{i_{k+1}}=R_{k+1}+\frac{d_{k+1}}{2} \delta_{i_{k+1}}, \quad i \in\{1,2\}
$$

where $R_{k+1}$ is the central point between $P_{1_{k+1}}^{*}$ and $P_{2_{k+1}}^{*}$ and $\boldsymbol{\delta}_{i_{k+1}}$ is the unitary vector from $R_{k+1}$ to $P_{i_{k+1}}^{*}$ (see Figure 4). The corresponding hand configuration $\boldsymbol{Q}_{k+1}=\left\{\boldsymbol{q}_{1_{k+1}}, \boldsymbol{q}_{2_{k+1}}\right\}$ is obtained from the target contact points $P_{1_{k+1}}$ and $P_{2_{k+1}}$ using the inverse kinematics (IK) of the fingers.

The manipulation procedure ends when any of the following four termination conditions is activated, two of them are associated with the orientation error $\varepsilon_{o}$, and the other two with the motion constraints:

- The orientation error is zero, i.e. the object has reached the desired orientation.

- The current object orientation is not improved during a predetermined number of iterations.

- The expected grasp at the computed contact points does not satisfy the friction constraints.

- The computed contact points do not belong to the workspace of the fingers.

If none of the termination conditions is activated, the hand is moved towards $\boldsymbol{Q}_{k+1}$ to make the fingers reach the desired target contact points $P_{1_{k+1}}$ and $P_{2_{k+1}}$. After the finger movements a new manipulation iteration begins.

\section{Recognition of the local curvature of the object}

The local curvature of the object is determined on-line applying a circular regression over a set of contact points obtained during the manipulation. The contact points have to be mapped from the reference frame $\mathcal{W}$ to a reference frame associated to the object $\mathcal{O}$, being the origin of $\mathcal{O}$ at the first contact point $P_{1}^{w}$ and the orientation coincident with that of $\mathcal{W}$, both at the initial blind grasp. The mapping of the contact points is performed using the information generated during the manipulation: the distance between contact points $d_{k}$, the rolled distances on the fingertip surfaces $L_{i_{k}}$ (computed from consecutive measurements of the contact points on the tactile sensor), and the object orientation $\gamma_{k}$. Given $L_{i_{k}}$, the mapped contact points $P_{i_{k}}^{o}$ belongs to two regions defined by circular paths with radius $L_{1_{k}}$ and $L_{2_{k}}$, centered on the contact points $P_{1_{0}}^{o}$ and $P_{2_{0}}^{o}$ respectively, i.e. the first contact points at the initial blind grasp, as shown in Figure 5. In order to improve legibility, the subscript $k$ is removed in the follow expressions. The circular paths are described by:

$$
\begin{gathered}
\left(P_{1_{x}}^{o}\right)^{2}+\left(P_{1_{y}}^{o}\right)^{2}=L_{1}^{2} \\
\left(P_{2_{x}}^{o}-d \cos \gamma\right)^{2}+\left(P_{2_{y}}^{o}-d \sin \gamma\right)^{2}=L_{2}^{2}
\end{gathered}
$$

Besides, the mapped points $P_{1_{k}}^{o}$ and $P_{2_{k}}^{o}$ must satisfy

$$
\begin{gathered}
d=\sqrt{\left(P_{2_{x}}^{o}-P_{1_{x}}^{o}\right)^{2}+\left(P_{2_{y}}^{o}-P_{1_{y}}^{o}\right)^{2}} \\
\left(P_{2_{y}}^{o}-P_{1_{y}}^{o}\right)=\left(P_{2_{x}}^{o}-P_{1_{x}}^{o}\right) \tan \gamma
\end{gathered}
$$

Equations (16), (17), (18) and (19) are solved for $P_{1_{x}}^{o}, P_{2_{x}}^{o}$, $P_{1_{y}}^{o}$ and $P_{2_{y}}^{o}$ resulting in:

$$
\begin{gathered}
P_{1_{x}}^{o}=P_{2_{x}}^{o}-\rho \\
P_{1_{y}}^{o}=P_{2_{y}}^{o}-\beta \\
P_{2_{x}}^{o}=\frac{-b \pm \sqrt{b^{2}-4 a c}}{2 a} \\
P_{2_{y}}^{o}=\sqrt{L_{2}^{2}-\left(P_{2_{x}}^{o}-d \cos \gamma\right)^{2}}+d \sin \gamma
\end{gathered}
$$

where

$$
\begin{aligned}
\rho= & \sqrt{\frac{d^{2}}{1+(\tan \gamma)^{2}}} \quad \beta=\tan \gamma \sqrt{\frac{d^{2}}{1+(\tan \gamma)^{2}}} \\
a & =(-2 d \sin \gamma+2 \beta)^{2}+(-2 d \cos \gamma+2 \rho)^{2} \\
b & =-2 d \cos \gamma(-2 d \sin \gamma+2 \beta)^{2} \\
& -2 \psi(-2 d \cos \gamma+2 \rho) \\
& +2 d \sin \gamma(-2 d \sin \gamma+2 \beta)(-2 d \cos \gamma+2 \rho) \\
c & =(d \cos \gamma)^{2}(-2 d \sin \gamma+2 \beta)^{2} \\
& +\psi^{2}-2 \psi d \sin \gamma(-2 d \sin \gamma+2 \beta) \\
& +(d \sin \gamma)^{2}(-2 d \sin \gamma+2 \beta)^{2} \\
& -L_{2}^{2}(-2 d \sin \gamma+2 \beta)^{2} \\
\psi= & L_{2}^{2}-L_{1}^{2}-(d \cos \gamma)^{2}-(d \sin \gamma)^{2}+\rho^{2}+\beta^{2}
\end{aligned}
$$

A detailed description of the procedure to identify the object shape is described in [12], although the information of the curvatures of the object is used here only to compute the target hand configurations instead of identifying the whole object model.

Let $S_{P_{i}^{o}}$ be the set of $m$ mapped contact points $P_{i}^{o}$ obtained during the manipulation process. The goal of the circular regression is to determine the circumferences $C_{i}^{o}$ 


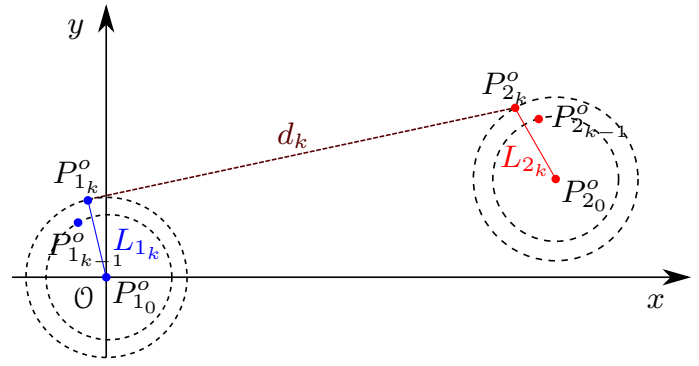

Fig. 5. Circular regions with radius $L_{1_{k}}$ and $L_{2_{k}}$, where the points belonging to the object surface are located.

with center point $\left(c_{i_{x}}^{o}, c_{i_{y}}^{o}\right)$ and radius $r_{c_{i}^{o}}$, so that the sum of the squared distances from each point in $S_{P_{i}^{o}}$ to $C_{i}^{o}$ is minimized, i.e. the points must be as close as possible to $C_{i}^{o}$ [17]. The assumption of circular shapes is based on the fact that "most objects in the world are made up of circular arcs and straight segments" [18]. Note that a straight segment can be seen as a circular arc with large enough radius. The obtained $C_{i}^{o}$ are mapped back to the absolute reference frame $\mathcal{W}$ by locating the center point $\left(c_{i_{x}}^{o}, c_{i_{y}}^{o}\right)$ at a distance $r_{c_{i}^{o}}$ from the contact point $P_{i_{k}}$ along the normal direction at $P_{i_{k}}$, as shown in Figure 3. Then $C_{i}^{w}$ is used to compute the virtual contact points $P_{i_{k+1}}^{*}$ as described in the manipulation procedure in Eq. (10) to Eq. (12) in Section III-B.

\section{REAL EXPERIMENTATION}

The Schunk Dexterous Hand (SDH2) was used for the experimental validation. The hand has three fingers with two dof each one, and an additional dof that allows the rotation of two fingers to work opposite to each other. A detailed description of the hand kinematics is presented in [19]. The hand has tactile sensors on the fingertips and the proximal phalanges, but only those on the fingertips are used in this work. Each sensor pad has 68 sensitive texels. Each texel registers a positive force up to $3 \mathrm{~N}$. Each tactile sensor provides the position of the barycenter of the actual contact region and the summation of the forces sensed over all the contacted texels. Then, in order to use the traditional punctual contact model [20], the barycenter of the contact region is used as the contact point position, and the summation of the forces on the contact region is considered as the resultant force applied on the contact point.

In each one of the following illustrative examples an object was brought close to the hand so that the hand closed the fingers to perform a blind grasp. The desired contact force was set to $F_{d}=4 \mathrm{~N}$. The friction coefficient was assumed to be $\mu=0.4$, which is lower than the real physical value considering that the fingertips are made of rubber. The constant $\lambda$ to adjust the distance between the contact points according to Eq. (14) was set to $\lambda=0.25 \mathrm{~mm}$. The constant $\Delta \gamma$ to change the object orientation was set to $\Delta \gamma=0.25$ degrees. The number of points to compute the local curvatures was set to $m=30$. The object was rotated first to a desired orientation $\gamma_{d}=5$ degrees and then to $\gamma_{d}=-5$ degrees. In order to illustrate the effect of

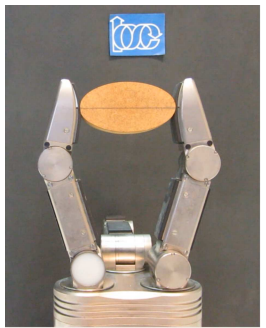

a)

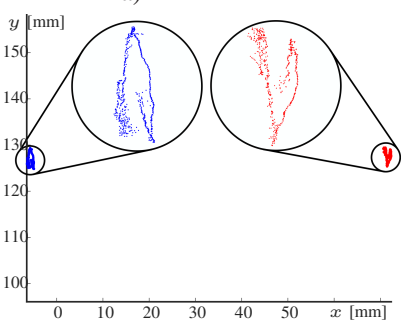

d)

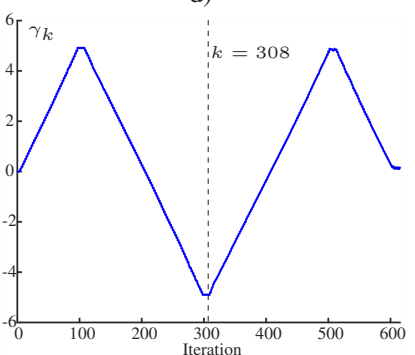

f)

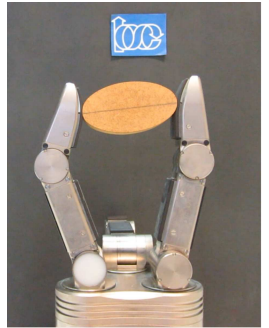

b)

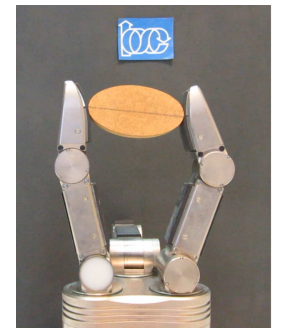

c)

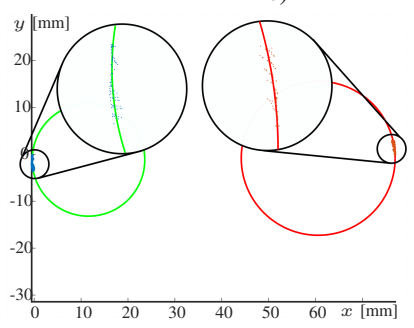

e)

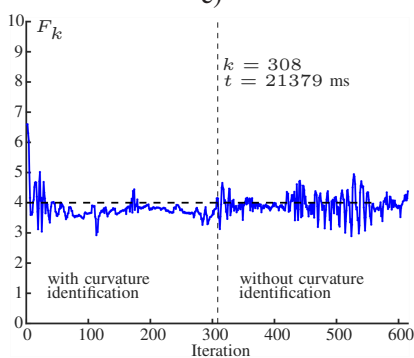

g)
Fig. 6. Manipulation of Object 1: a) Initial grasp; b) Object rotated 5 degrees; c) Object rotated -5 degrees; d) Resulting contact points $P_{i}^{w}$ with zoomed interest regions; e) Contact points $P_{i}^{o}$ and circumferences $C_{i}^{o}$ computed in the last circular regression, with zoomed interest regions; f) Evolution of the orientation $\gamma_{k}$ (in degrees); g) Evolution of the contact force $F_{k}$ (in Newtons), the horizontal dashed line indicates $F_{d}$ and the vertical dashed line the iteration in which the manipulation stops using the local curvatures.

using the local curvatures in the computation of the hand configurations, after these movements the object was rotated without using the local curvatures to $\gamma_{d}=5$ degrees and then to $\gamma_{d}=0$ degrees. In this is way the resulting contact forces in both cases can be directly compared.

Figure 6 shows the results of the manipulation of Object 1 . Figure $6 a$ to $6 \mathrm{c}$ show snapshots of the object in the initial grasp, rotated 5 degrees and rotated -5 degrees, respectively. Figure $6 \mathrm{~d}$ shows the contact points $P_{i}^{w}$. Figure 6e shows the contact points $P_{i}^{o}$ and the last circumferences $C_{i}^{o}$. Figure $6 \mathrm{f}$ shows the evolution of the object orientation $\gamma_{k}$, and Figure $6 \mathrm{~g}$ the contact force $F_{k}$, with and without the use of the local curvatures. Figure 7 shows the results of equivalent experiments using another three objects. A video of the experimental executions can be found in http://goo.gl/Wz7UH3.

Table I summarizes, for each object manipulation, the average contact force $\overline{F_{k}}$ (in Newtons), the corresponding variance $\sigma^{2}$ (in Newtons), the number of iterations required, and the average time per iteration (in ms), with and without using the computed local curvatures. The improvement of the contact force is indicated by its variance $\sigma^{2}$, when the 

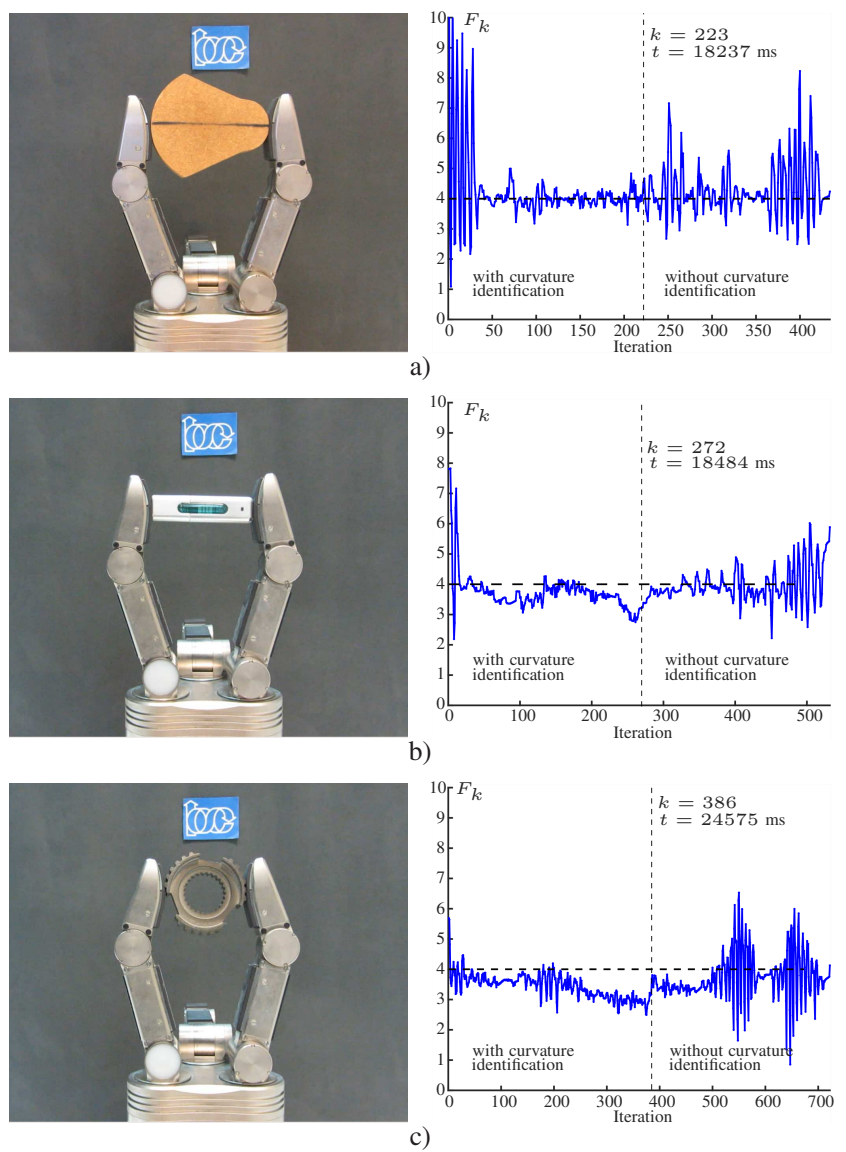

Fig. 7. a) to c) Manipulation of Objects 2, 3, and 4 respectively. Each case shows: (left) a snapshot of the initial grasp, and, (right) the evolution of the contact force $F_{k}$ (in Newtons), the horizontal dashed line indicates the desired contact force $F_{d}$ and the vertical dashed line the iteration in which the manipulation stops using the local curvatures.

TABLE I

EXPERIMENTAL DATA

\begin{tabular}{ccccc}
\hline & Object 1 & Object 2 & Object 3 & Object 4 \\
\hline \hline$\overline{F_{k}}$ with curvatures & 3.7284 & 4.0072 & 3.3971 & 3.6060 \\
\hline$\sigma^{2}$ & 0.0321 & 0.0826 & 0.1207 & 0.0953 \\
\hline Number of iterations & 308 & 223 & 272 & 386 \\
\hline Time per iteration & 69.41 & 81.78 & 67.96 & 63.67 \\
\hline$\overline{\overline{F_{k}}}$ without curvatures & 3.9288 & 4.2874 & 3.7385 & 3.9790 \\
\hline$\sigma^{2}$ & 0.1056 & 0.8161 & 0.5450 & 0.3222 \\
\hline Number of iterations & 306 & 210 & 259 & 335 \\
\hline Time per iteration & 73.48 & 76.06 & 69.17 & 69.97 \\
\hline
\end{tabular}

local curvature is used in the computation of the target hand configurations the contact force has a lower variance.

\section{SUMmARY AND FUtURE WORK}

This paper proposed an approach to improve the contact forces during the manipulation of unknown objects. It uses tactile and kinematic information generated during the manipulation to identifies on-line the local curvatures of the manipulated object and compute the hand configurations that allow a secure object rotation. The requirements for the implementation of the proposed approach in a robotic system are the knowledge of the hand kinematics, a position control of the fingertips and the availability of tactile information, as well as some compliance in the fingertip surfaces.

A natural extension of the proposed approach is the improvement of the contact forces during the optimization of other goals of the manipulation, as for instance, the hand configuration or the grasp quality, as well as the generalization to rotations in $3 \mathrm{D}$ space.

\section{REFERENCES}

[1] A. Bicchi. Hands for Dextrous Manipulation and Powerful Grasping: a Difficult Road Towards Simplicity. IEEE Transactions on Robotics and Automation, 16(6):652-662, 2000.

[2] M. Prats, A. Del Pobil, and P. J. Sanz. Robot Physical Interaction through the combination of Vision, Tactile and Force Feedback Applications to Assistive Robotics, volume 84 of Springer Tracts in Advanced Robotics. Springer, 2013.

[3] Z. Kappassov, J. A. Corrales, and V. Perdereau. Tactile sensing in dexterous robot hands review. Robotics and Autonomous Systems, $74: 195-220,2015$.

[4] A. Montaño and R. Suárez. Unknown object manipulation based on tactile information. In Proc. of IEEE/RSJ Int. Conf. on Intelligent Robots and Systems, pages 5642-5647, 2015.

[5] K. Tahara, S. Arimoto, and M. Yoshida. Dynamic object manipulation using a virtual frame by a triple soft-fingered robotic hand. In Proc. of IEEE Int. Conf. on Robotics and Automation, pages 4322-4327, 2010.

[6] Q. Li, M. Meier, R. Haschke, H. Ritter, and B. Bolder. Rotary object dexterous manipulation in hand: a feedback-based method. Int. Journal of Mechatronics and Automation, 3(1):36-47, 2013.

[7] S. K. Song, Jin P., and Y. H. Choi. Dual-fingered stable grasping control for an optimal force angle. IEEE Transactions on Robotics, 28(1):256-262, 2012.

[8] Z. Su, K. Hausman, Y. Chebotar, A. Molchanov, G. E. Loeb, G. S. Sukhatme, and S. Schaal. Force estimation and slip detection/classification for grip control using a biomimetic tactile sensor In 2015 IEEE-RAS 15th Int. Conf. on Humanoid Robots (Humanoids), pages 297-303, Nov 2015.

[9] F. Veiga, H. van Hoof, J. Peters, and T. Hermans. Stabilizing novel objects by learning to predict tactile slip. In IEEE/RSJ Int. Conf. on Intelligent Robots and Systems, pages 5065-5072, Sept 2015.

[10] R. Platt. Learning and Generalizing Control-Based Grasping and Manipulation Skills. PhD thesis, Univ. of Massachusetts, 2006.

[11] A. Bicchi, A. Marigo, and D. Prattichizzo. Dexterity through rolling: manipulation of unknown objects. In Proc. of IEEE Int. Conf. on Robotics and Automation, volume 2, pages 1583-1588, 1999.

[12] A. Montaño and R. Suárez. Object shape reconstruction based on the object manipulation. In Proc. of IEEE Int. Conf. on Advanced Robotics, pages 1-6, 2013.

[13] P. Toh, S. Huang, J. Lin, M. Bajzek, G. Zeglin, and N. Pollard. Dexterous telemanipulation with a multi-touch interface. In Proc. of IEEE-RAS Int. Conf. on Humanoid Robots, pages 270-277, 2012.

[14] C. Rosales, R. Suárez, M. Gabiccini, and A. Bicchi. On the synthesis of feasible and prehensile robotic grasps. In Proc. of IEEE Int. Conf. on Robotics and Automation, pages 550-556, may 2012.

[15] W. Shaw-Cortez, D. Oetomo, C. Manzie, and P. Choong. Tactilebased blind grasping: A discrete-time object manipulation controller for robotic hands. IEEE Robotics and Automation Letters, 3(2):1064 1071, April 2018.

[16] R. Ozawa, Suguru Arimoto, P.T.A. Nguyen, M. Yoshida, and Ji Hun Bae. Manipulation of a circular object in a horizontal plane by two finger robots. In Proc. of IEEE Int. Conf. on Robotics and Biomimetics, pages 517-522, 2004.

[17] N. Chernov. Circular and Linear Regression: Fitting Circles and Lines by Least Squares, volume 117 of Monographs on Statistics and Applied Probability. Chapman \& Hall / CRC, 2010.

[18] W. A. Perkins. A model-based vision system for industrial parts. IEEE Transactions on Computers, C-27(2):126-143, Feb 1978.

[19] A. Montaño and R. Suárez. Commanding the object orientation using dexterous manipulation. In Advances in Intelligent Systems and Computing, volume 418, pages 69-79. Springer Verlag, 2016.

[20] V.-D. Nguyen. Constructing Force- Closure Grasps. The Int. Journal of Robotics Research, 7(3):3-16, jun 1988. 